Case Report

\title{
A Case Report of a Bleeding Duodenal Gastro-Intestinal Stromal Tumor and its Emergent Management
}

\author{
Yagan Pillay *, Oladapo Mabadeje \\ Department of General Surgery, University of Saskatchewan, Saskatchewan, Canada
}

\section{Article history:}

Received: March 26, 2021

Revised: May 19, 2021

Accepted: May 20, 2021

\author{
*Corresponding Author: \\ Yagan Pillay \\ Department of General Surgery, University \\ of Saskatchewan, Health Sciences Building \\ 107 Wiggins Rd. B419, Saskatoon, S7N \\ OW8, Saskatchewan, Canada \\ E-mail: yagan2pillay@yahoo.ca \\ ORCID \\ Yagan Pillay \\ https://orcid.org/0000-0003-1254-3179
}

\begin{abstract}
A gastrointestinal stromal tumor (GIST) arising in the duodenum is a rare subtype of mesenchymal tumor. GISTs have a reported incidence of 11.9-19.6 per million population and duodenal GISTs make up just $5 \%$ of these tumors. Common presentation of duodenal GISTs is through an upper gastrointestinal bleed, of which, they are responsible for less than $1 \%$ of all gastrointestinal bleeding. In an elective setting, surgical management remains the mainstay of treatment. In this Case Report, the emergent management of a localized duodenal resection was performed by an acute care surgeon, in an unstable patient. The risk factors for malignancy include tumor size and a high mitotic cell index. Tumor recurrence is determined by tumor size, tumor rupture, high mitotic cell index, a non-gastric location and gastrointestinal bleeding.
\end{abstract}

Keywords: bleeding, duodenum, gastrointestinal stromal, gastrointestinal neoplasms, tumors

\section{Introduction}

A gastrointestinal stromal tumor (GIST) may arise from any part of the gastrointestinal tract but has a predilection for the stomach and the small bowel. Their origin has been reported to stem from interstitial cells of Cajal in the mesenchymal tissue [1,2], and $60-70 \%$ of GISTs are gastric in origin. Of these rare mesenchymal tumors, $5 \%$ arise from the $2^{\text {nd }}$ and $3^{\text {rd }}$ parts of the duodenum $[1,2]$. Common clinical presentations included bleeding and abdominal pain. Upper gastrointestinal bleeding caused by GISTs are rare with a reported incidence of less than $1 \%$ [1]. The tumor in this Case Report arose from the $3^{\text {rd }}$ part of the duodenum which is the $2^{\text {nd }}$ commonest location for duodenal GISTs.

\section{Case Report}

A male patient, 67 years of age, was referred to the acute care surgical team with epigastric abdominal pain. The pain was acute in onset and radiated to his back. There was no foodsymptom-pain association. This was his $1^{\text {st }}$ documented episode of this type of abdominal pain. His significant medical history included coronary artery disease, chronic kidney disease, diabetes, and hypertension. He was clinically stable, obese with a large pendulous abdomen, which made his physical examination difficult. His abdomen was soft and tender in the epigastrium but there was no rebound tenderness or guarding. There was no obvious intra-abdominal mass or abdominal wall hernia palpable and he was not jaundiced. The rectal examination was normal and he had brown stools. He had previously been prescribed non-steroidal anti-inflammatories for back pain and had a diagnosis of a peptic ulcer.

Laboratory investigations revealed leukocytosis and chronic renal failure. Liver enzymes and serum lipase levels were normal. No free air under the diaphragm was observed in the erect chest roentgenogram. An ultrasound to exclude cholelithiasis showed a heterogenous mass in the tail of the pancreas. The source of the lesion could not be ascertained using radiology and so a computerized tomography scan 
was performed. The scan showed a $7.1 \times 5 \mathrm{~cm}$ tumor in the $3^{\text {rd }}$ part of the duodenum (Figures $1 \mathrm{~A}, 1 \mathrm{~B}$, and $1 \mathrm{C}$ ). This tumor displayed features of heterogeneity in keeping with a radiological diagnosis of a GIST. The patient was then admitted for pain control and work up of his duodenal GIST. Twentyfour hours post-admission, he passed a large melaena stool and became hypotensive. He was resuscitated with intravenous fluids and packed red blood cells. A repeat hemoglobin $(\mathrm{Hb})$ was $62 \mathrm{~g} / \mathrm{L}$ compared with an initial $\mathrm{Hb}$ of $90 \mathrm{~g} / \mathrm{L}$ on admission 24 hours earlier. He remained hypotensive with a decreasing serial level of $\mathrm{Hb}$ despite an initial blood transfusion of 2 units of packed red cells. Clinically, his abdomen was soft and nontender with no palpable masses. He had no overt signs of peritonitis and a rectal exam revealed hematochezia as well as fresh melaena. The patient signed an informed consent for an emergency laparotomy with the understanding that he may require a total colectomy to stop his colonic bleeding if the source of his bleeding could not be ascertained.

He required extremely urgent surgery and was taken to the operating room whilst continuing the resuscitation with packed red blood cells and fresh frozen plasma. He was also started on an intravenous infusion of pantoprazole. The gastroscopy showed fresh blood in the duodenum with no obvious signs of a gastric or duodenal ulcer. The $3^{\text {rd }}$ part of the duodenum could not be reached to determine whether the GIST was the source of the bleeding so, a midline exploratory laparotomy was performed. Intraoperatively there was a large tumor attached to the $3^{\text {rd }}$ part of the duodenum (Figure $2 \mathrm{~A}$ ). The superior mesenteric vein that had draped over this tumor was mobilized away from the tumor without incident (Figure 2A). Through careful mobilization of the duodenal-jejunal flexure, the tumor on the antimesenteric border of the $3^{\text {rd }}$ part of the duodenum was carefully mobilized off the retroperitoneum (Figure 2B). The presence of blood visualized in the jejunum and ileum abated suspicion of colonic apathology. Proximal and distal tumor margins were ascertained and the $3^{\text {rd }}$ part of the duodenum was resected (Figures 2C and 2D). Duodenal- jejunal anastomosis was performed using a $60 \mathrm{~mm}$ linear stapler, however, the patient's body habitus and high body mass index led to difficulties. There were extensive adhesions over the $2^{\text {nd }}$ part of the duodenum and the decision was made to perform a retro-colonic gastro-jejunostomy to the posterior wall of the stomach. It was a side-to-side stapled anastomosis. Post-operatively the patient was managed in the Intensive Care Unit due to hypovolemic shock, metabolic acidosis, and inotropic support was required by the patient. He received 8 units of packed red cells and 10 units of fresh frozen plasma in total. He was extubated 24 hours later and was subsequently

(A)

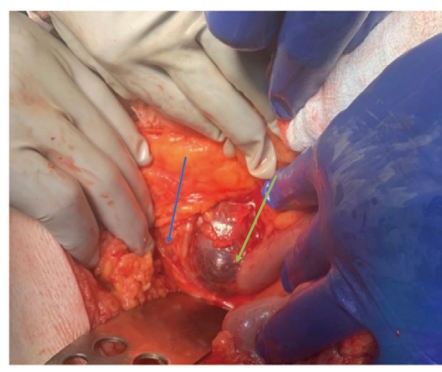

(B)

(C)

(D)

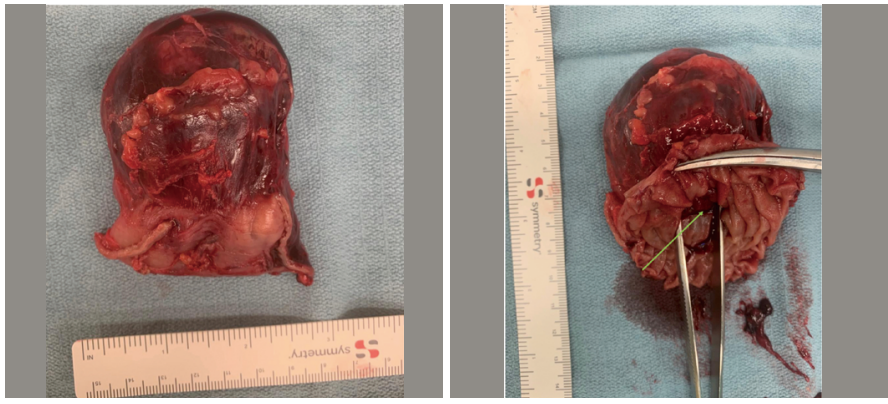

Figure 2. Surgery. (A) Tumor in situ arising from the $3^{\text {rd }}$ part of the duodenum (green arrow) with the inferior mesenteric vein mobilized medially (blue arrow); (B) Mobilization of the duodenal-jejunal flexure with the tumor in situ; (C) Resected specimen with stapled proximal and distal margins; (D) Duodenal opening of the tumor in the 3rd part of the duodenum with blood clot in situ (green arrow).
(A)

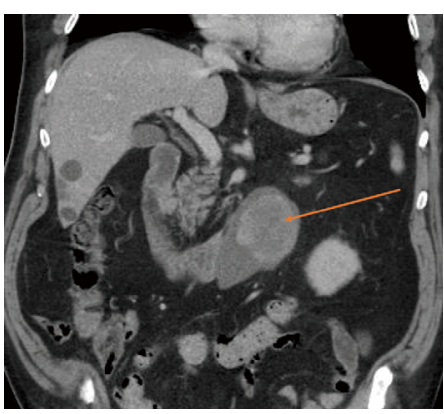

(B)

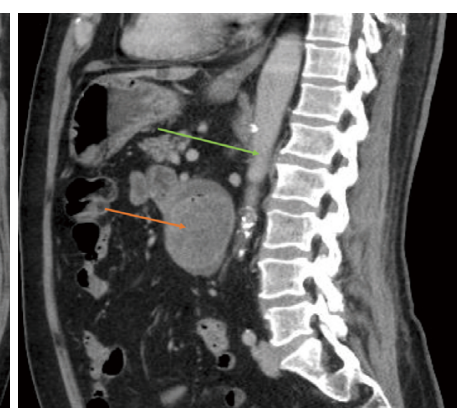

(C)

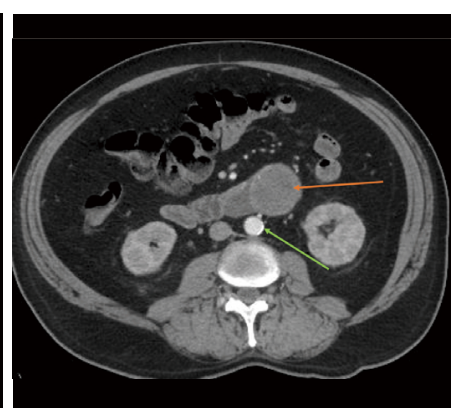

Figure 1. Radiology. (A) CT scan coronal view showing the tumor in the $3^{\text {rd }}$ part of the duodenum (orange arrow); (B) CT scan sagittal view showing the tumor (orange arrow) superior to the descending abdominal aorta (green arrow); (C) CT scan axial view showing the tumor in the $3^{\text {rd }}$ part of the duodenum (orange arrow) and superior to the abdominal aorta (green arrow). 
transferred onto the surgical ward. His $\mathrm{Hb}$ had stabilized at 86 $\mathrm{g} / \mathrm{dL}$. He was started on total parenteral nutrition as a result of a postoperative ileus. The patient commenced enteral nutrition 7 days after surgery and was discharged 5 days later, on a regular diet. He developed a partial wound dehiscence in the midline prior to discharge and was managed conservatively with Aquacel silver dressings, without the need for a negative pressure dressing. Pathology confirmed a GIST of the duodenum with clear margins (Figure 3). The patient's levels of CD117 and DOG1 as detected by immunohistochemistry, were highly positive.

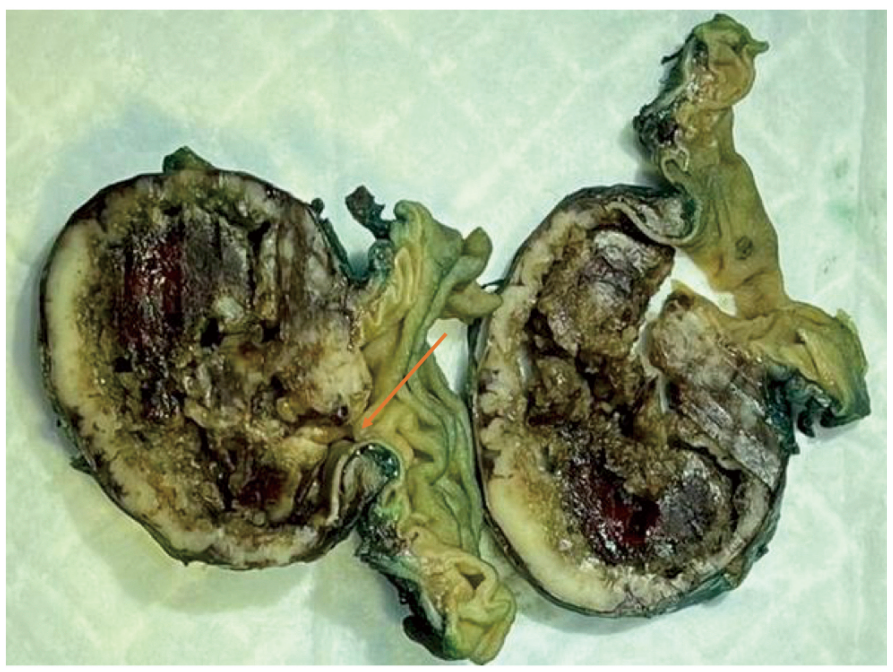

Figure 3. Pathology. Gross pathology of the resected tumor showing the tumor opening into the duodenum (orange arrow).

\section{Discussion}

GISTs are rare mesenchymal tumors with a reported incidence of 11-19.6 per million population [3]. Duodenal GISTs are extremely rare making up $5 \%$ of all reported cases with a predilection for the $2^{\text {nd }}$ or $3^{\text {rd }}$ part of the duodenum. It is usually seen in males in the $6^{\text {th }}$ decade of life [3].

Treatment involves surgical resection which can be quite complex, and may require a pancreaticoduodenectomy in tumors involving the ampulla of Vater, in the $2^{\text {nd }}$ part of the duodenum. Wide resection margins with a lymph node resection are not necessary because submucosal spread and lymph node involvement occurs in less than $5 \%$ of all GISTs [3].

Complimentary treatment strategies such as the use of Imatinib (a small molecule tyrosine kinase inhibitor) is often used in recurrent or metastatic tumors as neoadjuvant therapy. Imatinib is also effective in large primary tumors of the duodenum to enhance surgical resection [4]. If there is tumor regression a duodenal local resection, especially in the $2^{\text {nd }}$ part, becomes possible without the need for a pancreaticoduodenectomy and its attendant morbidity and mortality.

This patient presented with an acute gastrointestinal bleed requiring a red blood cell and plasma transfusion. There was no time to attempt alternative treatments or stop the bleeding endoscopically because the tumor was in the $3^{\text {rd }}$ part of the duodenum and out of reach for the endoscope. The health facility`s lack of interventional radiology meant an angiogram could not be attempted. Nevertheless, the patient's clinical instability would have precluded an angiogram. The size of the resected tumor $(7.1 \times 5 \mathrm{~cm})$ increased the suspicion of malignancy. Risk factors for malignancy include sonographic features such as a size greater than $4 \mathrm{~cm}$, echogenic foci, cystic spaces within the tumor, and an irregular extraluminal border [5]. Pathological features of a malignancy include a high mitotic index ( $>5$ per high powered field of view) [5].

Predilection for recurrence is determined by tumor size, tumor rupture, high mitotic index, and a non-gastric location [5]. The patient remains at high risk for recurrence due to the tumor size and location, and will continue to be treated with a tyrosine kinase inhibitor as part of his adjuvant therapy workup. GIST recurrence has a predilection for the peritoneum or liver. Long-term oncology follow-up is crucial to prevent a recurrence or distant metastasis. As a cause of an upper gastrointestinal bleed, the duodenal GIST is rare $(<1 \%)$. The etiology of the bleed involves mucosal ulceration and invasion of the submucosal blood vessels [6,7]. This is thought to contribute to tumor embolization and local recurrence or distant metastases. Gastrointestinal bleeding has now been postulated as an independent prognosticator for surgical recurrence $[6,7]$.

\section{Conclusion}

A rare etiology of upper gastrointestinal bleeding in the form of a duodenal GIST arising from the $3^{\text {rd }}$ part of the duodenum was presented in this Case Report. Haemostasias was achieved through surgical resection and a retro-colonic gastrojejunostomy in an acute care setting, in a clinically unstable patient.

\section{Conflicts of Interest}

The authors have no conflicts of interest to declare.

\section{Ethical Statement}

This research did not involve any human or animal experiment. 


\section{References}

[1] Santos AJ, Tojal A, Duarte L, Marques C, Pinheiro LF, Casimiro C. Asymptomatic large duodenal GIST - An incidental finding in abdominopelvic ultrasonography: A case report. Int J Surg Case Rep 2020;76:301-4.

[2] Beham A, Schaefer IM, Cameron S, von Hammerstein K, Füzesi L, Ramadori G, et al. Duodenal GIST: a single center experience. Int J Colorectal Dis 2013;28(4):581-90.

[3] Gu L, Khadaroo PA, Chen M, Qian H, Zhu H, Li X, et al. Surgical management and outcomes of duodenal gastrointestinal stromal tumors. Acta Gastroenterol Belg 2019;82(1):11-8.
[4] Hankiewicz-Ziołkowska K, Soboń M, Szylberg T, Rudziński J. Duodenal bulb tumor of unknown origin. Prz Gastroenterol 2014;9(6):365-70.

[5] Harris PS, Romano J, Russ KB, Shoreibah MG, Baig KRKK. Gastrointestinal Stromal Tumor: GIST Another Duodenal Ulcer. Ochsner J 2020;20(2):2368.

[6] Mahmoud S, Salman H, Salami M. A rare case report of locally recurrent hemorrhagic duodenal gastrointestinal stromal tumor: therapeutic challenges and review of prognostic indicators for recurrence. J Surg Case Rep 2020;2020(1):rjz365.

[7] Liu Q, Kong F, Zhou J, Dong M, Dong Q. Management of hemorrhage in gastrointestinal stromal tumors: a review. Cancer Manag Res 2018;10:73543. 